\title{
Animal-Based Remedies as Complementary Medicines in the Semi-Arid Region of Northeastern Brazil
}

\author{
Rômulo R. N. Alves, ${ }^{1}$ José A. A. Barbosa, ${ }^{1}$ Silene L. D. X. Santos, ${ }^{2}$ Wedson M. S. Souto, ${ }^{3}$ \\ and Raynner R. D. Barboza ${ }^{2}$ \\ ${ }^{1}$ Departamento de Biologia, Universidade Estadual da Paraíba, Avenida das Baraúnas, Campina Grande, Paraíba 58109-753, Brazil \\ ${ }^{2}$ Mestrado em Ciência e Tecnologia Ambiental, Universidade Estadual da Paraíba, Avenida das Baraúnas, Campina Grande, \\ Paraíba 58109-753, Brazil \\ ${ }^{3}$ Pós-Graduação em Desenvolvimento e Meio Ambiente (PRODEMA), Universidade Estadual da Paraíba, Avenida das Baraúnas, \\ Campina Grande, Paraíba 58109-753, Brazil
}

Correspondence should be addressed to Rômulo R. N. Alves, romulo_nobrega@yahoo.com.br

Received 6 February 2009; Accepted 3 August 2009

Copyright ( 2011 Rômulo R. N. Alves et al. This is an open access article distributed under the Creative Commons Attribution License, which permits unrestricted use, distribution, and reproduction in any medium, provided the original work is properly cited.

\begin{abstract}
Animals (and their derived products) are essential ingredients in the preparation of many traditional remedies. Despite its prevalence in traditional medical practices worldwide, research on medicinal animals has often been neglected in comparison to medicinal plant research. This work documents the medicinal animals used by a rural community in the semi-arid region, inserted in Caatinga Biome, where 66 respondents provided information on animal species used as medicine, body parts used to prepare the remedies and illnesses to which the remedies were prescribed. We calculated the informant consensus factor to determine the consensus over which species are effective for particular ailments, as well as the species use value to determine the extent of utilization of each species. We recorded the use of 51 animal species as medicines, whose products were recommended for the treatment of 68 illnesses. The informant consensus in the use of many specific remedies is fairly high, giving an additional validity to this folk medicine. Eight species not previously reported as having medicinal use were recorded. The local medicinal fauna is largely based on wild animals, including some endangered species. Given a high proportion of medicinal animals observed in the study area, it is logical to conclude that any conservation strategy should include access to modern health care.
\end{abstract}

\section{Introduction}

The use of a complete range of natural resources including plants, animals and mineral inorganic components is a common practice in traditional medicine. Animals and products derived from their organs have constituted part of the inventory of medicinal substances used in various cultures since ancient times [1-4].

Despite its prevalence in traditional medical practices worldwide, research on medicinal animals has often been neglected in comparison to medicinal plant research $[1,5]$. Emphasis was mostly put on medicinal plants because more different species as well as greater quantities are used than in the case for animals. Also, in many aspects plants are easier to collect, store and trade.

Recent publications have shown the importance of zootherapy in various socio-cultural environments around the world, and examples of the use of animal-derived remedies can currently be found in many urban, semi-urban and more remote localities in all parts of the world, particularly in developing countries [1,6-10]. In Brazil, many species of animals have been used for medicinal purposes since colonial times, with widely disseminated therapeutic alternatives available throughout the country [1,11-14]. Many people still use animal-derived medicines as an alternative or supplement to western health care [1, 15-17].

In Brazil, since the 1980s, various publications have shown the importance of zootherapy for traditional communities from distinct socio-cultural-environmental landscapes [1]. A recent review of the subject reported 290 different animal species being used in traditional folk medicine in Brazil [11]. This number is certainly underestimated since the amount of studies on the theme is very limited in number and locality. Most of what is known about medicinal animals 
comes from studies conducted in coastal areas and Amazon region (e.g., $[15,16,18-21])$. Comparatively, the leastacknowledged biomes are the Caatinga and Cerrado, two highly impacted ecosystems $[22,23]$. The Caatinga (semiarid) vegetation is a highly threatened biome covering a vast area in northeastern Brazil, and is the source of many littlestudied natural resources [22, 24-28].

The use of animals for medicinal purposes is part of a body of traditional knowledge which is increasingly becoming more relevant to discussions on conservation biology, public health policies, sustainable management of natural resources, biological prospection and patents $[5$, 29-33]. In northeastern Brazil, especially in the semi-arid region, animals and plants are widely used in traditional medicine and play a significant role in healing practices [27]. Zootherapies form an integral part of the local culture, and information about animals and their uses are passed from generation to generation through oral folk lore. In this context, the present study contributes to the documentation of the animals utilized as medicines among a group of inhabitants of the rural area in municipal district of Queimadas, inserted in Caatinga Biome, located in the state of Paraiba, Brazil.

\section{Methods}

2.1. Study Area. The present work was carried out in community of Castanho de Baixo located in municipal district of Queimadas Paraíba State, Northeastern Brazil (Figure 1). Queimadas covers an area of $409 \mathrm{~km}^{2}$ and is situated in the Agreste mesorregion from state cited, at the approximate geographical coordinates $7^{\circ} 21^{\prime} \mathrm{S}$ and $35^{\circ} 53^{\prime} \mathrm{W}$.

The climate is semi-arid and annual rainfall is between 400 and $800 \mathrm{~mm}$, with wettest period from November to April. The local vegetation is composed of a spiny caducifolious vegetation characteristic of this semi-arid region (Caatinga biome).

In Queimadas the total population is $\sim 39000$ of which, $56.2 \%$ live in the rural zone and $43.8 \%$ live in the city's urban area. The Human Development Index (HDI) is 0.595 (medium level development) [34]. In community studied (Castanho de Baixo), located in the rural zone, there are approximately 90 residences. The community of Queimada city is formed by typical Sertanejos people, descending from settlers families of the Agreste mesoregion of Paraíba.

About 130 years ago, the main existing forests in the mountains near the town provided excellent hunting, consequently leading the population to move there. These people began to populate the region, and since then still develop hunt activity in Castanho de Baixo area for food and commercialization. However, subsistence farming is the norm, primarily bean, corn, cassava, sweet potato, cattle, poultry, goats and sheep.

2.2. Procedures. Field research was conducted from September to November 2007 to February 2008. During the first contacts with the local population, we identified people with a specialized knowledge of medicinal animal use [15]. A specialist was defined as "a person recognized by the community as having deep knowledge about the use of animals in manufacturing remedies and in promoting cures". Information on the use of animals in traditional medicines was collected through interviews with 66 persons (18 men and 48 women), mainly from the elderly populations, who still retain the major portion of traditional knowledge in their respective communities. Additional interviewees were chosen from referral. Prior informed consent was obtained for all interviews conducted. The ethical approval for the study was obtained from the Ethics committee of Paraiba University State.

Survey data were gathered through individual interviews [35] and included local name of the animal used as remedy; parts used as medicine; conditions treated with the remedy; preparation and usage; restrictions of use; adverse effects; spiritual aspects linked to the use; use of live or dead animals; how animals were obtained; storage conditions; collection sites; gear used to collect the animals; efficacy of the remedies; traditional uses of the remedies in the community; how knowledge was acquired by the interviewees; reliance on animal-based remedies; why the interviewee used animalbased remedies.

Species' vernacular names were recorded as quoted by interviewees. Zoological material was identified with the aid of specialists, through (i) examination of voucher specimens donated by the interviewees; (ii) photographs of the animals or their parts, taken during interviews; (iii) vernacular names, with the aid of taxonomists familiar with the study areas" fauna. Voucher specimens and/or photographs were deposited at the Department of Systematics and Ecology, Federal University of Paraíba.

2.3. Data Analysis. The reputed therapeutic effects and ailments treated were grouped into 16 categories based on the classification used by the Centro Brasileiro de Classificação de Doenças (Brazilian Centre for the Classification of Diseases) [36] (Table 1).

To estimate the level of agreement between interviewees over which animals to use for each category, we calculated the informant consensus factor (ICF), adapted from Heinrich et al. [37] that quantifies the variability of animals used for each treatment, and therefore the consensus between practitioners. This factor estimates the relationship between the "number of use-reports in each category (nur) minus the number of taxa used $(n \mathrm{t})$ " and the "number of use-reports in each category minus 1 ". ICF is thus calculated using the following formula:

$$
\mathrm{ICF}=\frac{n \mathrm{ur}-n \mathrm{t}}{n \mathrm{ur}-1}
$$

The product of this factor ranges from 0 to 1 . A value near zero indicates a high variation in the use of species, if animals are chosen randomly, or if informants do not exchange information about their use. Values near 1 indicate a high intra-cultural consensus. 


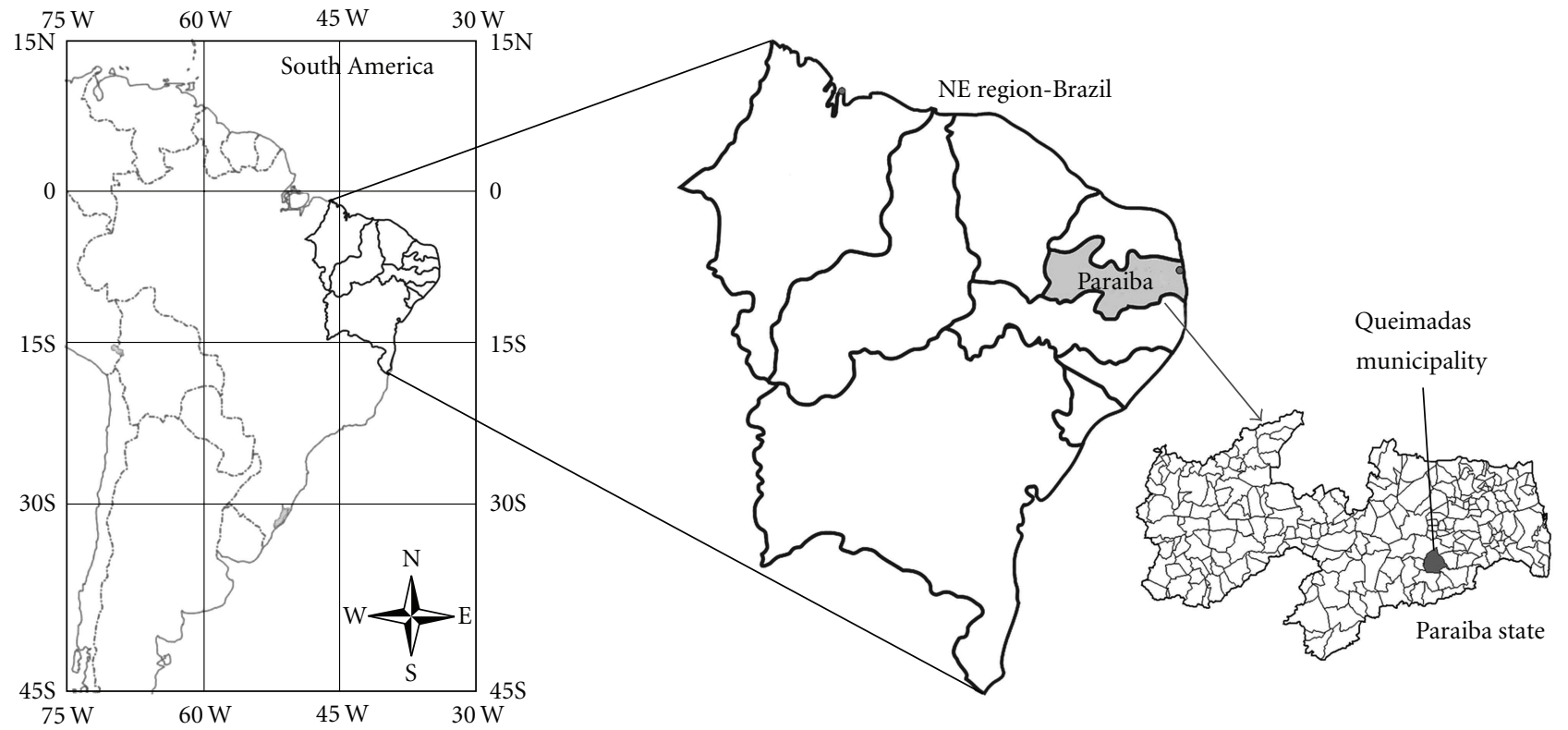

Figure 1: Map of study area, Municipality of Queimadas, Northeast Brazil.

The use-value (adapted from Phillips et al. [38]), a quantitative method demonstrating the relative importance of each species, was calculated as

$$
\mathrm{UV}=\frac{U}{n},
$$

where UV is the use-value of a species, $U$ the number of citations per species and $n$ is the number of informants.

Application of the use-value of each species is based objectively on the importance attributed by the informants and does not depend on the opinion of the researcher.

\section{Results}

The study documented 51 medicinal animals ( 42 vertebrates and 9 invertebrates). The reported species were distributed among 42 zoological families. Birds and mammals (both with 17 species) and reptiles and arthropods (both with six species) were best represented in terms of the number of species (Figure 2). Examples of animals used as medicine are shown in Figure 3.

The most commonly mentioned species were Gallus gallus domesticus-domestic chicken and Tupinambis merianae-lizard $(n=59)$, Ovis aries - domestic goat $(n=56)$, Atta cephalotes —ant $(n=47)$, Crotalus durissus — rattlesnake $(n=46)$, Euphractus sexcinctus - six-banded armadillo $(n=$ 43), Iguana iguana-common iguana $(n=43)$ and Bos taurus-domestic cattle $(n=42)$. The value use (VU) of zootherapeutic resources ranged from 0.03 to 0.893 . The species which attained the highest use-value were G. gallus domesticus (0.893), T. merianae (0.893), O. aries (0.848), C. thous (0.742), C. durissus (0.696), D. novemcinctus, I. iguana (0.651) and B. taurus (0.636) (Table 2).

Although $85 \%$ medicinal animals were reported in previous studies carried in Brazil, we identified eight species

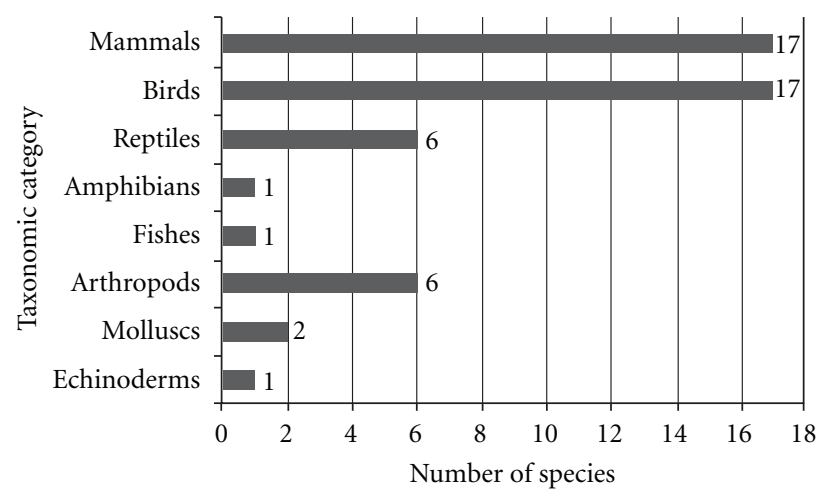

Figure 2: Number of animal species used as remedies per taxonomic category at the Municipality of Queimadas, Northeast Brazil.

not previously reported prescribed for treating a total of 18 illnesses: Iphigenia brasiliensis, Molossus molossus, Buteogallus urubutinga, Megalobulimus oblongus, Protonectarina sylveirae, Netta erythrophthalma, Columba picazuro and Acromyrmex landolti.

The local medicinal fauna was largely based on wild animals. Nevertheless, some domestic animal species are also used to produce materials for traditional medicine. These included the domestic cow (Bos taurus), domestic goat (Capra hircus), sheep (Ovis aries), dog (Canis lupus familiaris), ass (Equus asinus), horse (Equus caballus), domestic cat (Felis catus), pig (Sus scrofa), turkey (Meleagris gallopavo), helmeted guineafowl (Numida meleagris), "Codorna" (Coturnix coturnix), domestic chicken (Gallus gallus), Indian peafowl (Pavo cristatus) and Greater rhea (Rhea americana). 


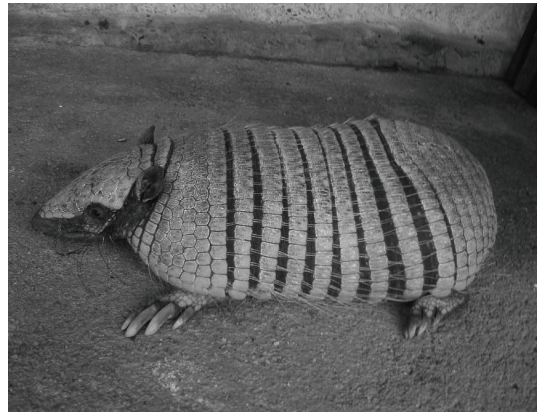

(a)

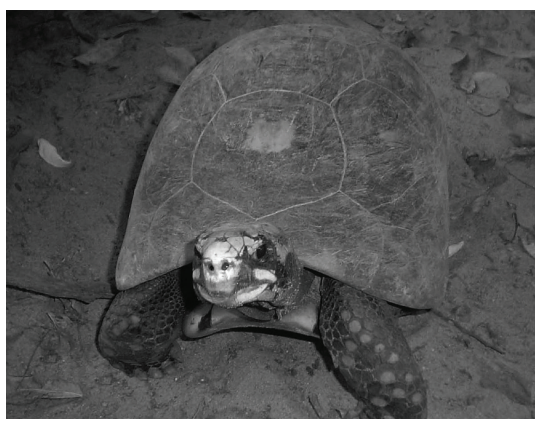

(c)

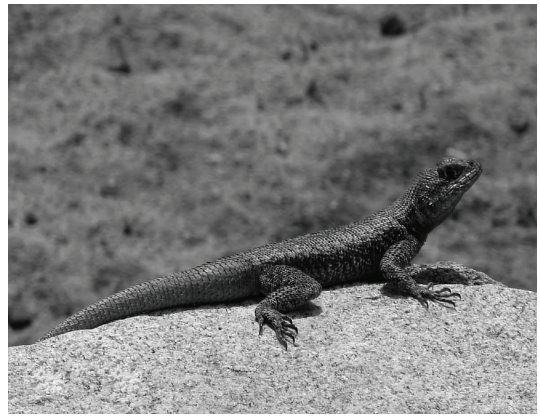

(e)

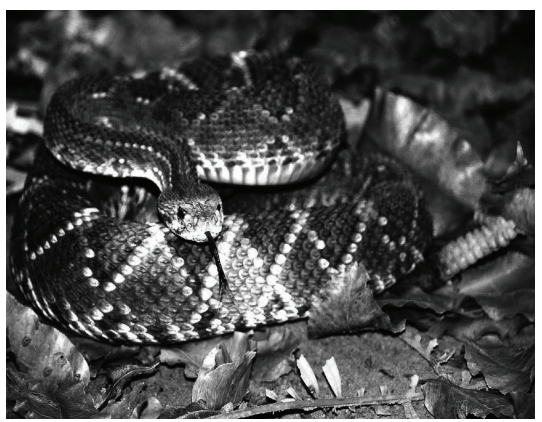

(g)

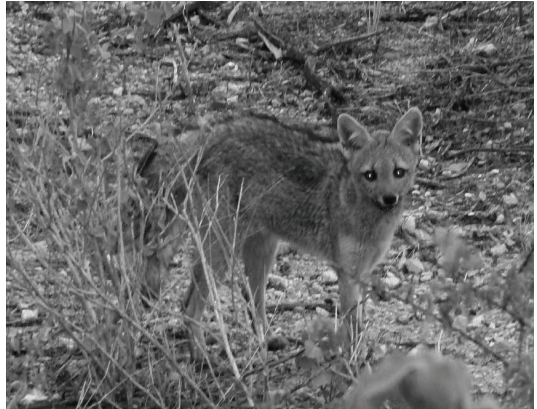

(b)

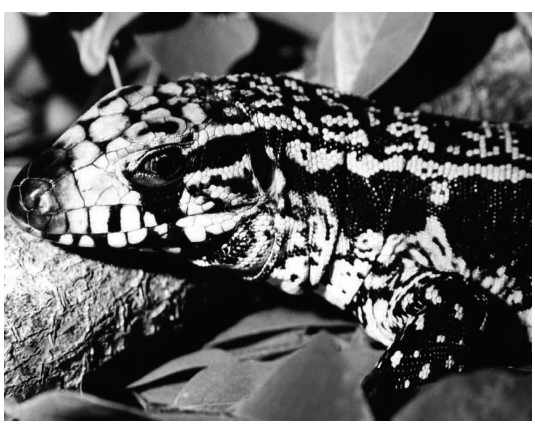

(d)

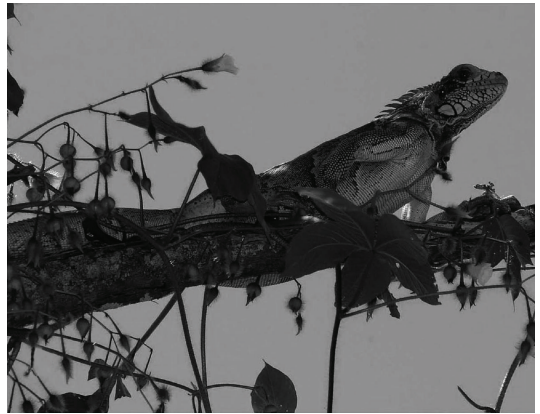

(f)

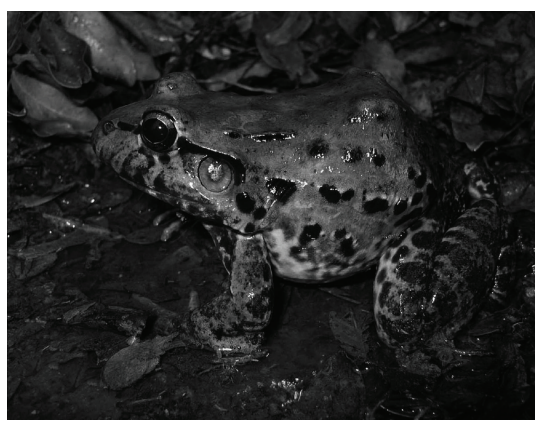

(h)

FIgURE 3: Examples of animals used as medicine in Northeast Brazil. (a) Euphractus sexcinctus (Photo: Wedson Souto), (b) Cerdocyon thous (Photo: Hélder Araújo), (c) Chelonoidis carbonaria (Photo: Rômulo Alves), (d) Tupinambis merianae (Photo: Yuri Lima), (e) Tropidurus hispidus (Photo: Washington Vieira), (f) Iguana iguana (Photo: Washington Vieira), (g) Crotalus durissus (Photo: Yuri Lima) and (h) Leptodactylus vastus (Photo: Claúdio Sampaio). 
TABLE 1: Categories of diseases treated with zootherapeutic remedies in surveyed area (Queimadas, Paraiba State), according to the Brazilian Centre for the Classification of Diseases.

\begin{tabular}{|c|c|c|}
\hline Categories & Diseases and illnesses mentioned by respondents & Total \\
\hline 1 & $\begin{array}{l}\text { Teething, inflammation, hoarseness, cracks in the sole of the feet, general pain, to assist children who take } \\
\text { longer than usual to start walking, children that speak with lateness, itching, problems of navel, "hard nerve" } \\
\text { and healing of umbilical cord of newborn baby }\end{array}$ & 11 \\
\hline 2 & $\begin{array}{l}\text { Erysipelas, oral mycosis, athlete's foot, measles, rubella, chickenpox, tuberculosis, warts, whooping cough and } \\
\text { mumps, moniliasis }\end{array}$ & 11 \\
\hline 3 & Asthma, bronchitis, effusion, catarrh, cough, flu, sore throat, sinusitis and tonsillitis & 9 \\
\hline 4 & Poor digestion & 1 \\
\hline 5 & Burns, wounds, muscle strain, luxation, distension and sprains & 6 \\
\hline 6 & Arthritis, arthrosis, backache, osteoporosis and rheumatism & 5 \\
\hline 7 & Hemorrhage, cardiac problems, thrombosis and cerebral hemorrhage & 4 \\
\hline 8 & Menstrual problems and urinary infection & 2 \\
\hline 9 & Alcoholism, snakes bite, insect bite, to suck a splinter out of skin or flesh, swelling and bleeding & 6 \\
\hline 10 & Boils, skin spots and acne, & 3 \\
\hline 11 & Problems in nerves & 1 \\
\hline 12 & Tumors & 1 \\
\hline 13 & Deafness, earache & 2 \\
\hline 14 & Diabetes, weakness and malnutrition & 3 \\
\hline 15 & Sexual impotence & 1 \\
\hline 16 & Conjunctivitis and pterygium & 2 \\
\hline Total & & 68 \\
\hline
\end{tabular}

(1) undefined diseases; (2) some infections and parasitic diseases; (3) respiratory system; (4) digestive system; (5) injuries, poisoning and other consequences of external causes; (6) osteomuscular system and conjunctive tissue; (7) circulatory system; (8) urinogenital system; (9) external causes of morbidity and mortality; (10) skin and subcutaneous tissue; (11) nervous system; (12) Symptoms, signs and abnormal findings from medical and laboratorial examination, not categorized in other part or section; (13) ear (middle and inner ear) and mastoid apophysis; (14) diseases of the endocrine glands, metabolism and nutrition; (15) mental and behavioral perturbations; (16) ophthalmological diseases.

Interviewees quoted the following animal byproducts used as remedies: flesh, bone, cartilage, skin, tail, fur, feather, tooth, nail, head, tongue, stomach, viscera, liver, bile, milk, fat, rattle (from rattlesnakes), spine, shell, abdomen and body secretions (see Table 2). Hard parts, such as teeth, nails, fish scales, bone and cartilage were generally sun dried, grated and crushed to powder, being then administered as tea or taken during meals. Fat, body secretion and oil were ingested or used as an ointment.

Zootherapeutic resources were used to cure about 68 ailments. As reflected in Table 3, the body system categories with the greatest number of species and treatment indications were respiratory system (27 species; 320 use-citations), the undefined illnesses (20 species; 126 use-citations) and the osteomuscular system and conjunctive tissue ( 9 species; 177 use-citations).

Table 3 summarizes the ICFs for the 16 ailment categories recorded, showing the different levels of cultural consensus. The highest ICF values were for digestive and nervous system $(\mathrm{ICF}=1)$ and for diseases of circulatory system $(0.97)$. We reported that C. coturnix (UV: 0.56 ) was the most frequently used species for mental and behavioral perturbations and $P$. sylveirae (0.515) for circulatory system. The fourth highest ICF value (0.95) was recorded for diseases of osteomuscular system and conjunctive tissue, which was most often treated using the fox (C. thous) (UV: 0.74) and C. durissus (UV: 0.69).

Over $75.5 \%$ of animal species were reported to cure more than one ailment. For instance, products of the domestic chicken (G. gallus domesticus) were used to treat at least 11 illnesses and I. iguana was used to treat at least nine illnesses (Table 2). On the other hand, different animal species were sometimes used to treat the same illness. For instance, products obtained from 18 different species were used to treat asthma.

Animals provided the raw materials for remedies prescribed clinically and are also used in the form of amulets and charms in magic, religious rituals and ceremonies. Some respondents associated the use and efficacy of some remedies to popular beliefs locally known as "simpatias". As examples they mentioned that animals' parts were used as amulets against diseases and that the person receiving a given treatment should not know the source else the effect would cease. Examples of "simpatias" include the use of teeth of "Prea" (Cavia aperea) as an amulet to treat teething; scrubbing a child's knee on "Donkey" (Equus asinus) footprints in order to make the child "walk early"; and "rattlesnake" (C. durissus) rattle as amulet to avoid serpents bites. 
TABLE 2: Animal taxa recorded as having medicinal properties.

\begin{tabular}{|c|c|c|c|c|}
\hline Family/species/local name & $\begin{array}{l}\text { Number of } \\
\text { mentions }\end{array}$ & $\begin{array}{c}\text { Relative } \\
\text { importance } \\
\text { (UV) }\end{array}$ & $\begin{array}{l}\text { Part used and way of } \\
\text { administration }\end{array}$ & Disease (or illness) \\
\hline \multicolumn{5}{|l|}{ Echinoderms } \\
\hline \multicolumn{5}{|l|}{ Oreasteridae } \\
\hline $\begin{array}{l}\text { Oreaster reticulatus (Linnaeus, } \\
\text { 1758)-Starfish, "estrela-do-mar" }\end{array}$ & 7 & 0.106 & Whole animal $(3,5)$ & Asthma \\
\hline \multicolumn{5}{|l|}{ Mollusks } \\
\hline \multicolumn{5}{|l|}{ Megalobulimidae } \\
\hline $\begin{array}{l}\text { Megalobulimus oblongus (Mïller, } \\
\text { 1774)-clam }\end{array}$ & 4 & 0.06 & Whole animal (5) & Asthma \\
\hline \multicolumn{5}{|l|}{ Donacidae } \\
\hline $\begin{array}{l}\text { Iphigenia brasiliensis (Lamarck, } \\
\text { 1818)-“concha-do-mar" }\end{array}$ & 3 & 0.045 & Shell (8) & Teething \\
\hline \multicolumn{5}{|l|}{ Arthropods } \\
\hline \multicolumn{5}{|l|}{ Apidae } \\
\hline $\begin{array}{l}\text { Apis mellifera (Linnaeus, 1758) } \\
\text { "abelha" (Africanized honey bee) }\end{array}$ & 22 & 0.333 & Honey $(4,18)$ & $\begin{array}{l}\text { Bronchitis, "catarrh in the } \\
\text { chest", coughs, influenza, } \\
\text { sore throat, sinusitis, } \\
\text { tonsillitis, hoarseness, } \\
\text { tuberculosis and whooping } \\
\text { cough }\end{array}$ \\
\hline \multicolumn{5}{|l|}{ Blattidae } \\
\hline \multirow[t]{2}{*}{$\begin{array}{l}\text { Periplaneta americana (Linnaeus, } \\
\text { 1758)-American cockroach, "barata" }\end{array}$} & 14 & 0.212 & Offal (9) & Earache \\
\hline & & & Whole animal (3) & Asthma \\
\hline \multicolumn{5}{|l|}{ Formicidae } \\
\hline $\begin{array}{l}\text { Acromyrmex landolti (Emery, } \\
\text { 1980)-"saúva" }\end{array}$ & 11 & 0.166 & Whole animal (5) & Asthma \\
\hline Atta cephalotes-"tanajura" & 47 & 0.712 & Abdomen (4) & $\begin{array}{l}\text { Sore throat, "catarrh in the } \\
\text { chest", cough and } \\
\text { hoarseness }\end{array}$ \\
\hline \multicolumn{5}{|l|}{ Termitidae } \\
\hline $\begin{array}{l}\text { Nasutitermes macrocephalus (Silvestri, } \\
\text { 1903)-Termite, "cupim” }\end{array}$ & 39 & 0.590 & Whole animal (16) & $\begin{array}{l}\text { Bronchitis, "catarrh in the } \\
\text { chest" coughs, influenza, } \\
\text { sore throat, sinusitis, } \\
\text { tonsillitis and hoarseness }\end{array}$ \\
\hline \multicolumn{5}{|l|}{ Vespidae } \\
\hline $\begin{array}{l}\text { Protonectarina sylveirae (Saussure, } \\
\text { 1854)-“marimbondo-preto" } \\
\text { "marimbondo-mosquito" }\end{array}$ & 34 & 0.515 & Nest $(19,5)$ & $\begin{array}{l}\text { Mumps, hemorrhage, } \\
\text { blooding and menstrual } \\
\text { problems }\end{array}$ \\
\hline \multicolumn{5}{|l|}{ Fishes } \\
\hline \multicolumn{5}{|l|}{ Gadidae } \\
\hline $\begin{array}{l}\text { Gadus morhua, Linnaeus, } \\
1758^{\mathrm{VU}} \text {-Atlantic cod "Bacalhau” }\end{array}$ & 3 & 0.045 & Fat $(2,6,4)$ & Backache and rheumatism \\
\hline \multicolumn{5}{|l|}{ Amphibians } \\
\hline \multicolumn{5}{|l|}{ Hylidae } \\
\hline Leptodactylus vastus, Lutz, 1930-"Jia" & 18 & 0.272 & Fat $(4)$, meat $(1,12)$ & Sore throat \\
\hline \multicolumn{5}{|l|}{ Reptiles } \\
\hline \multicolumn{5}{|l|}{ Chelidae } \\
\hline $\begin{array}{l}\text { Mesoclemmys tuberculata } \\
\text { (Luederwaldt, 1926)-Tuberculate } \\
\text { toadhead turtle, "cágado", } \\
\text { "cágado-d'água" }\end{array}$ & 38 & 0.575 & Fat $(2,6,17)$ & $\begin{array}{l}\text { Column pain backache, } \\
\text { rheumatism, swell, } \\
\text { furuncles and tumors }\end{array}$ \\
\hline
\end{tabular}


TABle 2: Continued.

\begin{tabular}{|c|c|c|c|c|}
\hline Family/species/local name & $\begin{array}{l}\text { Number of } \\
\text { mentions }\end{array}$ & $\begin{array}{l}\text { Relative } \\
\text { importance } \\
\text { (UV) }\end{array}$ & $\begin{array}{l}\text { Part used and way of } \\
\text { administration }\end{array}$ & Disease (or illness) \\
\hline \multicolumn{5}{|l|}{ Crotalidae } \\
\hline \multirow[t]{2}{*}{$\begin{array}{l}\text { Crotalus durissus (Linnaeus, } \\
1758)^{\mathrm{DD} / I I I} \text {-Neotropical rattlesnake, } \\
\text { "cascavel" }\end{array}$} & \multirow[t]{2}{*}{46} & \multirow[t]{2}{*}{0.696} & Fat $(2,6,4)$ & $\begin{array}{l}\text { Rheumatism, pains in } \\
\text { general, backache, and } \\
\text { inflammation }\end{array}$ \\
\hline & & & “Maracá” (rattle) (8) & Snake bite \\
\hline \multicolumn{5}{|l|}{ Iguanidae } \\
\hline \multirow[t]{2}{*}{$\begin{array}{l}\text { Iguana iguana (Linnaeus, } \\
\text { 1758) } \\
\text { "cam/II_Common iguana, }\end{array}$} & \multirow[t]{2}{*}{43} & \multirow[t]{2}{*}{0.651} & Fat $(2,6,4,9)$ & $\begin{array}{l}\text { Rheumatism, pains in } \\
\text { general, column pain, sore } \\
\text { throat, earache, arthritis, } \\
\text { osteoarthritis, furuncles } \\
\text { and tumors }\end{array}$ \\
\hline & & & Bones $(3,14)$ & $\begin{array}{l}\text { Rheumatism, arthritis and } \\
\text { osteoarthritis }\end{array}$ \\
\hline \multicolumn{5}{|l|}{ Teiidae } \\
\hline $\begin{array}{l}\text { Tupinambis merianae (Duméril \& } \\
\text { Bibron, 1839) } \\
\text { "tejulII - Lizard," "teju", }\end{array}$ & 59 & 0.893 & Fat $(4,9,2,6,17)$ & $\begin{array}{l}\text { Sore throat, earache, } \\
\text { "catarrh in the chest", } \\
\text { coughs, influenza, } \\
\text { hoarseness, tumor and } \\
\text { swelling }\end{array}$ \\
\hline \multicolumn{5}{|l|}{ Testudinidae } \\
\hline \multirow[t]{2}{*}{$\begin{array}{l}\text { Chelonoidis carbonaria (Spix, } \\
\text { 1824) } \\
\text { "jabutiI-Red-footed tortoise, }\end{array}$} & \multirow[t]{2}{*}{9} & \multirow[t]{2}{*}{0.136} & Fat $(2,6,17)$ & $\begin{array}{l}\text { Furuncles, tumors and } \\
\text { pains in general }\end{array}$ \\
\hline & & & Whole animal (11) & Asthma \\
\hline \multicolumn{5}{|l|}{ Tropiduridae } \\
\hline \multirow[t]{3}{*}{$\begin{array}{l}\text { Tropidurus hispidus (Spix, } \\
\text { 1825)-“lagartixa" (Lizard) }\end{array}$} & \multirow[t]{3}{*}{34} & \multirow[t]{3}{*}{0.515} & Offal (6) & $\begin{array}{l}\text { Erysipelas, chilblain, warts, } \\
\text { skin spots and cracks in the } \\
\text { sole of the feet }\end{array}$ \\
\hline & & & Tail (5) & Asthma \\
\hline & & & $\begin{array}{l}\text { Whole animal }(1,5 \text {, } \\
6)\end{array}$ & $\begin{array}{l}\text { Asthma, chilblain, warts } \\
\text { and skin spots }\end{array}$ \\
\hline \multicolumn{5}{|l|}{ Birds } \\
\hline \multicolumn{5}{|l|}{ Accipitridae } \\
\hline $\begin{array}{l}\text { Buteogallus urubitinga (J. F. Gmelin, } \\
\text { 1788)-"gavião-cauã" }\end{array}$ & 3 & 0.045 & Bones $(3,14)$ & $\begin{array}{l}\text { Backache, Column pain } \\
\text { and rheumatism }\end{array}$ \\
\hline \multicolumn{5}{|l|}{ Anatidae } \\
\hline $\begin{array}{l}\text { Anas platyrhynchos Linnaeus, } \\
\text { 1758-"patos" (Domestic duck) }\end{array}$ & 27 & 0.409 & Eggs $(4,12)$ & $\begin{array}{l}\text { Male impotence, and } \\
\text { weakness }\end{array}$ \\
\hline $\begin{array}{l}\text { Netta erythrophthalma } \\
\text { (Wied-Neuwied, 1833)-“paturi” }\end{array}$ & 19 & 0.287 & Eggs $(4,12)$ & $\begin{array}{l}\text { Male impotence and } \\
\text { weakness }\end{array}$ \\
\hline \multicolumn{5}{|l|}{ Cathartidae } \\
\hline \multirow[t]{2}{*}{$\begin{array}{l}\text { Coragyps atratus (Bechstein, } \\
\text { 1793)-"urubu" (Black vulture) }\end{array}$} & \multirow[t]{2}{*}{18} & \multirow[t]{2}{*}{0.272} & $\begin{array}{l}\text { Liver and feather }(3 \text {, } \\
5,15)\end{array}$ & Asthma, alcoholism \\
\hline & & & Whole animal (16) & Tuberculosis \\
\hline \multicolumn{5}{|l|}{ Columbidae } \\
\hline $\begin{array}{l}\text { Columba livia (Gmelin, } \\
\text { 1789)-"pombo" (Rock pigeon) }\end{array}$ & 6 & 0.090 & Meat (12) & Asthma \\
\hline $\begin{array}{l}\text { Columba picazuro, Temminck, } \\
\text { 1813-"asa-branca" }\end{array}$ & 5 & 0.075 & Whole animal $(1,12)$ & $\begin{array}{l}\text { Sore throat, tonsillitis, } \\
\text { bronchitis and hoarseness }\end{array}$ \\
\hline $\begin{array}{l}\text { Leptotila rufaxilla (Richard \& Bernard, } \\
\text { 1792)-Gray-fronted dove, "juriti" }\end{array}$ & 8 & 0.121 & Gizzard (10) & Pterygium \\
\hline
\end{tabular}


TABle 2: Continued.

\begin{tabular}{|c|c|c|c|c|}
\hline Family/species/local name & $\begin{array}{l}\text { Number of } \\
\text { mentions }\end{array}$ & $\begin{array}{c}\text { Relative } \\
\text { importance } \\
\text { (UV) }\end{array}$ & $\begin{array}{l}\text { Part used and way of } \\
\text { administration }\end{array}$ & Disease (or illness) \\
\hline \multicolumn{5}{|l|}{ Corvidae } \\
\hline $\begin{array}{l}\text { Cyanocorax cyanopogon (Wied, } \\
\text { 1821)-White-naped jay, "cancão" }\end{array}$ & 21 & 0.318 & Whole animal (11) & Asthma \\
\hline \multicolumn{5}{|l|}{ Falconidae } \\
\hline $\begin{array}{l}\text { Herpetotheres cachinnans (Linnaeus, } \\
1758)^{\mathrm{LR} / \mathrm{II}} \text { “acauã" (Laughing falcon) }\end{array}$ & 3 & 0.045 & Whole animal (1) & $\begin{array}{l}\text { Sore throat, tonsillitis and } \\
\text { hoarseness }\end{array}$ \\
\hline \multicolumn{5}{|l|}{ Meleagrididae } \\
\hline \multirow[t]{2}{*}{$\begin{array}{l}\text { Meleagris gallopavo Linnaeus, } \\
\text { 1758-turkey, "peru" }\end{array}$} & \multirow[t]{2}{*}{13} & \multirow[t]{2}{*}{0.196} & Fat $(17,2,6)$ & $\begin{array}{l}\text { Furuncles, tumors and } \\
\text { cracks in the sole of the feet }\end{array}$ \\
\hline & & & Feather $(3)$ & Asthma \\
\hline \multicolumn{5}{|l|}{ Numinidae } \\
\hline \multirow[t]{2}{*}{$\begin{array}{l}\text { Numida meleagris Linnaeus, } \\
\text { 1758-Helmeted Guineafowl, "Guiné” }\end{array}$} & \multirow[t]{2}{*}{9} & \multirow[t]{2}{*}{0.136} & Fat $(6,17)$ & Tumors and furuncles \\
\hline & & & Whole animal (1) & Pertussis \\
\hline \multicolumn{5}{|l|}{ Phasianidae } \\
\hline $\begin{array}{l}\text { Coturnix coturnix (Linnaeus, } \\
\text { 1758)-“codorna" }\end{array}$ & 37 & 0.560 & Eggs $(4,12)$ & $\begin{array}{l}\text { Male impotence, urinary } \\
\text { infection and weakness }\end{array}$ \\
\hline \multirow[t]{4}{*}{$\begin{array}{l}\text { Gallus gallus domesticus (Linnaeus, } \\
\text { 1758)-Domestic chicken, "galinha" }\end{array}$} & \multirow[t]{4}{*}{59} & \multirow[t]{4}{*}{0.893} & Fat $(4,2,6,17,9)$ & $\begin{array}{l}\text { Bronchitis, "catarrh in the } \\
\text { chest", coughs, influenza, } \\
\text { sore throat, sinusitis, } \\
\text { tonsillitis, swelling, } \\
\text { furuncles, tumors, and } \\
\text { earache }\end{array}$ \\
\hline & & & Gizzard (5) & Poor digestion \\
\hline & & & Eggs (6) & $\begin{array}{l}\text { Problems of navel, Healing } \\
\text { of umbilical cord of } \\
\text { newborn baby }\end{array}$ \\
\hline & & & $\begin{array}{l}\text { The whole animal } \\
\text { (13) }\end{array}$ & $\begin{array}{l}\text { It is long of the child to } \\
\text { begin to speak to assist } \\
\text { children who take longer } \\
\text { than usual to start walking }\end{array}$ \\
\hline $\begin{array}{l}\text { Pavo cristatus Linnaeus, 1758-"pavão", } \\
\text { Indian peafowl }\end{array}$ & 16 & 0.242 & Featherses (3) & Asthma \\
\hline \multicolumn{5}{|l|}{ Psitacidae } \\
\hline $\begin{array}{l}\text { Amazona aestiva (Linnaeus, } \\
\text { 1758)-“papagaio”" }\end{array}$ & 5 & 0.075 & Feces $(5,6)$ & $\begin{array}{l}\text { Asthma, skin spots and } \\
\text { acne }\end{array}$ \\
\hline \multicolumn{5}{|l|}{ Rheidae } \\
\hline \multirow[t]{2}{*}{$\begin{array}{l}\text { Rhea americana (Linnaeus, 1758) } \\
\text { LR/II_Greater rhea, "ema" }\end{array}$} & \multirow[t]{2}{*}{4} & \multirow[t]{2}{*}{0.060} & Eggs $(4,12)$ & Weakness and malnutrition \\
\hline & & & Fat $(2,6)$ & Pains in general \\
\hline \multicolumn{5}{|l|}{ Tyrannidae } \\
\hline $\begin{array}{l}\text { Fluvicola nengeta (Linnaeus, } \\
\text { 1766)-Masked water-tyrant, } \\
\text { "lavandeira" }\end{array}$ & 12 & 0.181 & The whole animal (3) & Asthma \\
\hline \multicolumn{5}{|l|}{ Mammalia } \\
\hline \multicolumn{5}{|l|}{ Bovidae } \\
\hline \multirow[t]{4}{*}{$\begin{array}{l}\text { Bos taurus Linnaeus, 1758-"gado" } \\
\text { (cattle) (cow) }\end{array}$} & \multirow[t]{4}{*}{42} & \multirow[t]{4}{*}{0.636} & Bone marrow $(1,12)$ & $\begin{array}{l}\text { Problems in the nerves and } \\
\text { weakness }\end{array}$ \\
\hline & & & "Fel" (bile) $(2,6)$ & $\begin{array}{l}\text { Suck a splinter out of skin } \\
\text { or flesh }\end{array}$ \\
\hline & & & $\begin{array}{l}\text { Excretion urinary }(2, \\
4)\end{array}$ & $\begin{array}{l}\text { Chilblain, diabetes and } \\
\text { conjunctivitis }\end{array}$ \\
\hline & & & Penis $(3,5)$ & Asthma \\
\hline
\end{tabular}


Table 2: Continued.

\begin{tabular}{|c|c|c|c|c|}
\hline Family/species/local name & $\begin{array}{c}\text { Number of } \\
\text { mentions }\end{array}$ & $\begin{array}{l}\text { Relative } \\
\text { importance } \\
\text { (UV) }\end{array}$ & $\begin{array}{l}\text { Part used and way of } \\
\text { administration }\end{array}$ & Disease (or illness) \\
\hline \multirow[t]{2}{*}{$\begin{array}{l}\text { Capra hircus Linnaeus, 1758-"cabra", } \\
\text { "bode" (Domestic goat) }\end{array}$} & 33 & 0.5 & Milk $(4,12)$ & Weakness and malnutrition \\
\hline & & & Hair (3) & Asthma \\
\hline $\begin{array}{l}\text { Ovis aries (Linnaeus, 1758)-“carneiro” } \\
\text { (Sheep) }\end{array}$ & 56 & 0.848 & Suet (2) & $\begin{array}{l}\text { Hard nerve, suck a splinter } \\
\text { out of skin or flesh, cardiac } \\
\text { problems, inflammation, } \\
\text { sprains and swelling }\end{array}$ \\
\hline \multicolumn{5}{|l|}{ Canidae } \\
\hline $\begin{array}{l}\text { Canis lupus familiaris (Linnaeus, } \\
\text { 1758)-"cachorro" (Domestic dog) }\end{array}$ & 19 & 0.287 & Feces $(5)$ & Measles and chicken pox \\
\hline $\begin{array}{l}\text { Cerdocyon thous (Linnaeus, 1766) }{ }^{\mathrm{LR} / \mathrm{II}} \text { - } \\
\text { "raposa" (Fox) }\end{array}$ & 49 & 0.742 & Fat (2) & $\begin{array}{l}\text { Arthritis, osteoarthritis, } \\
\text { osteoporosis, rheumatism, } \\
\text { column pain, sprain and } \\
\text { swelling }\end{array}$ \\
\hline \multicolumn{5}{|l|}{ Caviidae } \\
\hline \multirow[t]{2}{*}{ Cavia aperea Erxleben, 1777-“Preá” } & 16 & 0.242 & Fat $(2,6)$ & $\begin{array}{l}\text { Crack in the feet cracks in } \\
\text { the sole of the feet, }\end{array}$ \\
\hline & & & $\begin{array}{l}\text { Teeth (8), head (1) } \\
\text { and whole animal (1) }\end{array}$ & Teething \\
\hline \multirow[t]{2}{*}{$\begin{array}{l}\text { Kerodon rupestris (Wied-Neuwied, } \\
\text { 1820)-“Mocó" (Rock cavy) }\end{array}$} & 15 & 0.227 & Fat (9) & Deafness \\
\hline & & & Estomach (5) & Thrombosis and effusion \\
\hline \multicolumn{5}{|l|}{ Dasypodidae } \\
\hline $\begin{array}{l}\text { Dasypus novemcinctus, (Linnaeus, } \\
\text { 1758)-“tatu” }\end{array}$ & 43 & 0.651 & Tail (9) & Deafness and earache \\
\hline \multicolumn{5}{|l|}{ Didelphidae } \\
\hline $\begin{array}{l}\text { Didelphis albiventris (Lund, } \\
\text { 1840)-“timbú” (Common opossum) }\end{array}$ & 8 & 0.121 & Fat $(2,6)$ & $\begin{array}{l}\text { Arthritis, osteoarthritis, } \\
\text { osteoporosis, backache, } \\
\text { rheumatism and sprains }\end{array}$ \\
\hline \multicolumn{5}{|l|}{ Equidae } \\
\hline \multirow[t]{2}{*}{$\begin{array}{l}\text { Equus asinus Linnaeus, } \\
\text { 1758-"jumento" (Asino) }\end{array}$} & 37 & 0.560 & Milk (4) & $\begin{array}{l}\text { Weakness and malnutrition } \\
\text { to assist children who take }\end{array}$ \\
\hline & & & $\begin{array}{l}\text { "Trace" (footprints) } \\
\text { (6) }\end{array}$ & $\begin{array}{l}\text { longer than usual to start } \\
\text { walking }\end{array}$ \\
\hline $\begin{array}{l}\text { Equus caballus (Linnaeus, } \\
\text { 1758)-“cavalo" (Horse) }\end{array}$ & 7 & 0.106 & Hair (7) & Warts \\
\hline \multicolumn{5}{|l|}{ Felidae } \\
\hline $\begin{array}{l}\text { Felis catus Linnaeus, 1758, 1775-"gato" } \\
\text { (Domestic cat) }\end{array}$ & 4 & 0.06 & The whole animal (6) & Rubella \\
\hline \multicolumn{5}{|l|}{ Hominidae } \\
\hline $\begin{array}{l}\text { Homo sapiens Linnaeus, 1758-"gente" } \\
\text { (People) }\end{array}$ & 11 & 0.166 & $\begin{array}{l}\text { Excretion Urinary }(2, \\
6,4)\end{array}$ & $\begin{array}{l}\text { Itching, insect bite, } \\
\text { conjunctivitis and diabetes }\end{array}$ \\
\hline \multicolumn{5}{|l|}{ Mustelidae } \\
\hline $\begin{array}{l}\text { Conepatus semistriatus (Boddaert, } \\
\text { 1785)-"tacaca" (Striped hog-nosed } \\
\text { skunk) }\end{array}$ & 11 & 0.166 & Fat $(2,4)$ & Arthritis and osteoporosis \\
\hline \multicolumn{5}{|l|}{ Suidae } \\
\hline \multirow[t]{2}{*}{$\begin{array}{l}\text { Sus scrofa (Linnaeus, 1758)-"porco" } \\
\text { (Pig) }\end{array}$} & 28 & 0.424 & Fat $(2,6)$ & $\begin{array}{l}\text { Crack in the feet, burns and } \\
\text { wounds }\end{array}$ \\
\hline & & & “Fel” (bile) $(2,6)$ & $\begin{array}{l}\text { Suck a splinter out of skin } \\
\text { or flesh }\end{array}$ \\
\hline
\end{tabular}


Table 2: Continued.

\begin{tabular}{|c|c|c|c|c|}
\hline Family/species/local name & $\begin{array}{l}\text { Number of } \\
\text { mentions }\end{array}$ & $\begin{array}{c}\text { Relative } \\
\text { importance } \\
\text { (UV) }\end{array}$ & $\begin{array}{l}\text { Part used and way of } \\
\text { administration }\end{array}$ & Disease (or illness) \\
\hline \multicolumn{5}{|l|}{ Trichechidae } \\
\hline $\begin{array}{l}\text { Trichechus inunguis (Nattrer, 1883) }{ }^{\mathrm{VU} / \mathrm{I}} \text { - } \\
\text { "peixe-boi" (Amazonian manatee) }\end{array}$ & 5 & 0.075 & Fat $(2,4)$ & $\begin{array}{l}\text { Wounds, inflammation, } \\
\text { sprain, muscle strain, suck } \\
\text { a splinter out of skin or } \\
\text { flesh, arthritis, } \\
\text { osteoarthritis, osteoporosis } \\
\text { and rheumatism }\end{array}$ \\
\hline \multicolumn{5}{|l|}{ Molossidae } \\
\hline $\begin{array}{l}\text { Molossus molossus (Pallas, 1766), } \\
\text { Pallas' free-tailed bat (Bat) }\end{array}$ & 2 & 0.03 & The whole animal (3) & Asthma \\
\hline
\end{tabular}

TABLE 3: ICF categorized by medicinal use for corporal ailment.

\begin{tabular}{|c|c|c|c|c|c|}
\hline Ailment category & Species & $\begin{array}{l}\text { All species } \\
(\%)\end{array}$ & Use citation & $\begin{array}{c}\text { All use } \\
\text { citations }(\%)\end{array}$ & ICF \\
\hline Undefined illnesses & 20 & 37.73 & 126 & 8.09 & 0.848 \\
\hline Some infections and parasitic diseases & 9 & 17.64 & 106 & 6.95 & 0.923 \\
\hline Respiratory system & 25 & 49.01 & 299 & 19.61 & 0.919 \\
\hline Digestive system & 1 & 1.88 & 38 & 2.44 & 1 \\
\hline Injuries, poisoning and other consequences of external causes & 8 & 15.09 & 117 & 7.51 & 0.939 \\
\hline Osteomuscular system and conjunctive tissue & 9 & 16.98 & 177 & 11.37 & 0.954 \\
\hline Circulatory system & 2 & 3.77 & 49 & 3.14 & 0.979 \\
\hline Urinogenital system & 2 & 3.77 & 6 & 0.38 & 0.800 \\
\hline External causes of morbidity and mortality & 8 & 15.09 & 123 & 7.90 & 0.942 \\
\hline Skin and subcutaneous tissue & 8 & 15.09 & 119 & 7.64 & 0.940 \\
\hline Nervous system & 1 & 1.88 & 8 & 0.51 & 1 \\
\hline $\begin{array}{l}\text { Symptoms, signs, and abnormal findings from medical and } \\
\text { laboratorial examination, not categorized in other part or section }\end{array}$ & 7 & 13.20 & 81 & 5.20 & 0.925 \\
\hline Ear (middle and inner ear) and mastoid apophysis & 6 & 11.76 & 69 & 4.52 & 0.926 \\
\hline Diseases of the endocrine glands, metabolism and nutrition & 8 & 15.09 & 97 & 6.26 & 0.927 \\
\hline Mental and behavioral perturbations & 3 & 5.66 & 83 & 5.33 & 0.975 \\
\hline Ophthalmological diseases & 3 & 5.66 & 26 & 1.67 & 0.920 \\
\hline
\end{tabular}

\section{Discussion}

Our study revealed that 51 medicinal animals were being used in surveyed area, indicating very rich ethnomedical knowledge of the local area. Eight of the identified species have not previously been reported as having medicinal use.

The high use of vertebrates reported in our study is in line with other studies $[1-3,7,14,15,39-46]$. With regards to habitat type, nearly all animals recorded were from terrestrial habitats (49 species) - a reflection of principal habitat types found in the surveyed area, located in the semi-arid region. Similarly, Adeola [3] has shown that in Nigeria the utilization of wildlife was related to the ecological zone in which the people lived, and to the relative abundance of species in each zone. This finding demonstrates the importance of local biodiversity in furnishing folk medicines, in agreement with Alves and Rosa [16] who observed that faunal composition, accessibility and availability directly influence the types of zootherapeutic items used in any given region.

The most of the medicinal animals were native to the semi-arid region, with the exception of Oreaster reticulatus, Gadus morhua, Trichechus inunguis and Iphigenia brasiliensis. These species were found at public markets in Campina Grande city, situated $22 \mathrm{~km}$ from the surveyed area, where marine species are traded [46]. The use of marine and estuarine species in the middle of the semi-arid region can be explained by the existence of established trade routes for medicinal animals throughout the north and northeast of 
Brazil $[9,15,24,40,42,46-50]$. As pointed out by Alves et al. [46], the trade routes of medicinal animals traverse not only municipalities, but also Brazilian states.

Different parts of a single species provided the raw materials to prepare different remedies, which were prescribed to treat various diseases. The possibility of using various remedies for the same ailment is popularly valued, as it renders an adaptation to the availability/accessibility of the animals possible [15]. On the other hand, different species were sometimes used to treat the same illness. This strategy is important because many Caatinga species have a marked seasonality [28].

Zootherapeutical products are mainly used for the treatment of respiratory system diseases. A similar trend in relation to medicinal animals was found in cities of north and northeast of Brazil, where the two most frequently quoted categories of use referred to gastrointestinal and respiratory diseases $[15,16,24,40,46]$.

Animals provide the raw materials for remedies used to treat physical and/or spiritual diseases. The use of some zootherapeutical resources is associated with popular beliefs $[1,2,15-17,24,46]$. Those links should be taken into consideration when interpreting results of field surveys and when designing public health programs for communities where traditional medicine is used. In some cases, integrative approaches encompassing an understanding of traditional cultural views and insights concerning the cause, dissemination and treatment of a disease might be required to effectively treat it [15].

Some of the medicinal animals that are used by the local people in present study find mention in ancient medicinal literature in Brazil. Examples of species used in Brazil since colonial times are: I. iguana, C. durissus, Coragyps atratus and Bos taurus $[1,42,51]$. This verification corroborates what Almeida [51] described as the "high capability of reproduction of zootherapeutic practices in Brazil". The persistent use of animal-based medicines suggests that substances of therapeutic value not yet known by science may be present.

Despite their importance, analyses of the therapeutic use of animals and animal parts have been neglected, when compared with plants. However, within complementary and alternative medicine (CAM), zootherapy has been explored from the viewpoint of evidence-based [52], because the literature appears "glutted" with products derived from plants [53]. Both sources of natural products provide extensive sources of new CAM approaches that may emerge as important for future applications, including compounds isolated from marine microorganisms and phytoplankton, green algae, brown algae, red algae, fungi and certain well-known marine and terrestrial animals: sponges, coelenterates, bryozoans, mollusks, tunicates echinoderms, earthworms and leeches $[54,55]$.

It is widely accepted that folk or traditional medicinal uses (ethnomedical information) of biological resources indicate the presence of a biologically active constituent (s). In other words, folk or traditional medicinal uses represent "leads" that could shortcut the discovery of modern medicines [15, 16]. According to McGirk [56], Brazilian scientists are studying a type of frog that is used to cure intestinal illnesses by members of the Yawanawa Indian tribes. Indeed, amphibians have provided compounds capable of being turned to therapeutic advantage. Peptides extracted from the scraped secretions of Phyllomedusa bicolor, for instance, are used in the treatment of depression, stroke, seizures and cognitive loss in ailments such as Alzheimer's disease [57]. Some of these compounds are important tools for biochemical research or as new leads for the development of anticancer or antiviral drugs [58]. Regarding fish, several compounds have been extracted and these are employed as remedies in the official medicine [59]. Finkl [60], for example, refers to Eptatretus stoutii, Dasyatis sabina, and Taricha sp. as sources of cardiac stimulants, antitumors and analgesic, respectively. Oily fish, like cod, herring, salmon and turbot, have a great medicinal value to human beings due to a polyunsaturated compound known as OMEGA-3. This substance helps the prevention of arthritis [61]. The presence of an anticoagulant system in the plasma of Atlantic salmon (Salmo salar L.) and rainbow trout (Oncorhynchus mykiss Walbaun) has been confirmed, which supports similarities with the protein $\mathrm{C}$ anticoagulant system in mammals [62]. Geckos, frogs and other various insects are used in many Asiatic Materia Medica; meloid ("blister") beetles and leeches were listed for a long time in Western Pharmacopoeias and maggots has been recently listed in the US Pharmacopoeia $[63,64]$.

Clinical studies are lacking for most $[5,65]$. However, in the absence of scientific knowledge, consensus among practitioners in the use of particular remedies for particular ailments and level of usage may indicate effectiveness of use [66]. This can potentially be followed up by clinical studies and aid in the development of pharmaceutical drugs [67]. Among the species quoted by interviewees in the present study, some have previously been tested and their therapeutic effects evaluated. Murari et al. [68] provided evidence showing that $P$. cristatus feather extract inhibited hyaluronidase and proteolytic enzyme activities caused by the venom of Vipera russelii, Naja naja and Trimeresurus malabaricus, demonstrating a mechanism by which it could neutralize venom toxicity. Bisset [69] showed that the analgesic substances in the venom of species belonging to the families Viperidae, Crotalidae and Elapidae are more potent than morphine. The analgesic properties of venoms of some snake species, including $C$. durissus terrificus venom, have been demonstrated in humans and in experimental animal models [70-74]. Honey produced by honeybees (Apis mellifera) has both bacteriostatic and bactericidal effect [75]. Park et al. [76] isolated milk proteins of lactoforicine type from $B$. taurus with activity against bacteria. Cow urine has also been found to increase phagocytosis by macrophages and thus is sought helpful in prevention and control of bacterial infections. Besides this, cow urine has antioxidant property which protects DNA damage due to mitomycin-C-induced chromosomal aberrations [77].

In Brazil, most of the medicinal animals used are collected from the wild $[1,14-18,40,46,77,78]$. This same trend was observed in this study, where $28(77.7 \%)$ of the species traded are sourced from the wild. Nevertheless, many 
domestic animal species are also used to produce materials for traditional medicine.

The common use of domestic species may result from the ease of obtaining those animals and/or decline in wild fauna populations due to overhunting and loss of habitat. Human activities such as slash and burn agriculture, goat and cattle raising and extensive subsistence hunting are thought to be causing severe environmental impoverishment and a loss of biodiversity in the Caatinga [23].

This study also identified nine species of medicinal animals on either the IUCN Red List of Threatened Species (http://www.iucnredlist.org/), CITES list (Convention on International Trade in Endangered Species of Wild Fauna and Flora (http://www.cites.org/eng/resources/species.html), Brazil's official list of endangered species [79] or the National List of species of aquatic invertebrates and fishes endangered, overexploited or threatened by exploitation [80].

Unscrupulous usage of animal products in traditional medicines has led to many undesirable consequences including illegal trafficking of animal products. Pocking of animals for their medicinally important parts has brought many of the wild species under the red data book, for a possibility of their extinction. Many genera and species of wild animals have been considered at the brim of extinction as a consequence of overexploitation either of their own or of their habitat $[2,29]$. Anyinam [81] pointed out that, like the current spasm of plant and animal species extinction, the practitioners of ethnomedicine appear to be at a greater risk of extinction than even forests and other biomes. Environmental degradation affects users of traditional medicine both by limiting their access to the resources traditionally used and by diminishing the knowledge base in their community upon which traditional medicine is constructed.

The medicinal use of animals must be considered together with other anthropogenic pressures $[1,2]$. Rapid reduction in natural resources as a consequence to the expanded urbanization, global warming and reduced natural habitat posed a considerable threat to the sustainability of traditional medicine. Being completely dependent upon natural resources like herbs, minerals and animal products, traditional medicine would possibly rank first in order of extinction of heritage if an alternative way is not considered well in time [29].

There is a need to increase our understanding of the biology and ecology of species commonly used as remedies to better assess the impacts of harvesting them (for medicinal or other purposes) on their wild populations. Medicinal species whose conservation status is in question should receive urgent attention and aspects such as habitat loss and alteration should be discussed in connection with present and future medicinal uses [1].

The idea of sustainability in traditional medicine can well be traced through different cultures and societies with different notions [82-84]. Zootherapeutic activity, if properly managed, can be compatible with an environmental conservation program in which the use of natural resources can and must occur in such a way that human needs and protection of biodiversity are guaranteed [15]. The therapeutic indications of wild animals and plants and domestic or cultivated species overlapped in many cases. This aspect opens the possibility of, where suitable, replacing the use of threatened species with others in traditional medicine recipes $[1,2]$. Using domestic species does not represent a threat to the ecosystem and on top of that the use of such species may lead to large-scale use of natural products, as long as their efficacy is confirmed.

Besides being influenced by cultural aspects, the relations between humans and biodiversity in the form of zootherapeutic practices are conditioned by the social and economic relations between humans themselves. We would suggest further studies on these traditional remedies to confirm the presence of any bioactive compounds and also include this traditional knowledge into the strategies of conservation and management of faunistic resources for sustainable use.

Using animal products as components of bioprospecting has implications for medicines, the environment, economy, public health and culture [5, 85-89]. There is a great necessity to educate the local population and healers to adopt conservation measures as necessary, so that over collection of such species will not lead to their extinction in their territory, which signifies the loss of their source medicinal material. Given the high proportion of medicinal animals observed in the study area, it is logical to conclude that any conservation strategy would improve health care, particularly for rural communities with limited access to modern health facilities.

\section{Acknowledgments}

The authors would like to thank Universidade Estadual da Paraíba (UEPB)/Programa de Incentivo à Pós-Graduação e Pesquisa (PROPESQ)-011/2008 for the financial support that made the field surveys possible; Programa Institucional de Bolsas de Iniciação Científica-(PIBIC)/Conselho Nacional de Desenvolvimento Científico e Tecnológico (CNPq)/UEPB for the graduate fellowship conceded to the second author and Washington Vieira for his assistance with the figures. Special thanks are due to all interviewees, who kindly shared their knowledge with us.

\section{References}

[1] R. R. N. Alves, I. L. Rosa, and G. G. Santana, "The role of animal-derived remedies as complementary medicine in Brazil," BioScience, vol. 57, no. 11, pp. 949-955, 2007.

[2] R. R. N. Alves, W. L. Da Silva Vieira, and G. G. Santana, "Reptiles used in traditional folk medicine: conservation implications," Biodiversity and Conservation, vol. 17, no. 8, pp. 2037-2049, 2008.

[3] M. O. Adeola, "Importance of wild animals and their parts in the culture, religious festivals, and traditional medicine, of Nigeria," Environmental Conservation, vol. 19, no. 2, pp. 125134, 1992.

[4] E. Lev, "Traditional healing with animals (zootherapy): medieval to present-day Levantine practice," Journal of Ethnopharmacology, vol. 85, no. 1, pp. 107-118, 2003.

[5] R. R. N. Alves and I. L. Rosa, "Why study the use of animal products in traditional medicines?" Journal of Ethnobiology and Ethnomedicine, vol. 1, article 5, 2005. 
[6] M. M. Mahawar and D. P. Jaroli, "Traditional zootherapeutic studies in India: a review," Journal of Ethnobiology and Ethnomedicine, vol. 4, article 17, 2008.

[7] P. E. Vázquez, R. M. Méndez, O. G. R. Guiascón, and E. J. N. Piñera, "Uso medicinal de la fauna silvestre en los Altos de Chiapas, México," Interciencia, vol. 31, no. 7, pp. 491-499, 2006.

[8] N. Walston, "An overview of the use of Cambodia's wild plants and animals in traditional medicine systems," TRAFFIC Southeast Asia, Indochina, 2005.

[9] A. A. C. Pinto and C. B. Maduro, "Produtos e subprodutos da medicina popular comercializados na cidade de Boa Vista, Roraima," Acta Amazônica, vol. 33, pp. 281-290, 2003.

[10] O. A. Sodeinde and D. A. Soewu, "Pilot study of the traditional medicine trade in Nigeria," Traffic Bulletin, vol. 18, pp. 35-40, 1999.

[11] R. R. N. Alves, "Animal-based remedies as complementary medicine in Brazil," Forschende Komplementarmedizin, vol. 15, no. 4, pp. 226-227, 2008.

[12] J. G. W. Marques, Pescando Pescadores: etnoecologia abrangente no baixo São Francisco Alagoano, NUPAUB/USP, São Paulo, Brazil, 1995.

[13] F. D. B. P. Moura and J. G. W. Marques, "Folk medicine using animals in the Chapada Diamantina: incidental medicine?" Ciência \& Saúde Coletiva, vol. 13, no. 2, pp. 2179-2188, 2008.

[14] F. S. Ferreira, A. V. Brito, S. C. Ribeiro, A. A. F. Saraiva, W. O. Almeida, and R. R. N. Alves, "Animal-based folk remedies sold in public markets in Crato and Juazeiro do Norte, Ceará, Brazil," BMC Complementary and Alternative Medicine, vol. 9, article 17, 2009.

[15] R. R. N. Alves and I. L. Rosa, "From cnidarians to mammals: the use of animals as remedies in fishing communities in NE Brazil," Journal of Ethnopharmacology, vol. 107, no. 2, pp. 259 276, 2006.

[16] R. R. N. Alves and I. L. Rosa, "Zootherapeutic practices among fishing communities in North and Northeast Brazil: a comparison," Journal of Ethnopharmacology, vol. 111, no. 1, pp. 82-103, 2007.

[17] R. R. N. Alves, N. A. Léo Neto, G. G. Santana, W. L. S. Vieira, and W. O. Almeida, "Reptiles used for medicinal and magic religious purposes in Brazil," Applied Herpetology, vol. 6, no. 3, pp. 257-274, 2009.

[18] L. Branch and M. F. Silva, "Folk medicine in Alter do Chão, Pará, Brasil," Acta Amazônica, vol. 13, pp. 737-797, 1983.

[19] N. Figueiredo, "Os 'bichos' que curam: os animais e a medicina 'folk' em Belém do Pará," Boletim do Museu Paraense Emílio Göeldi, vol. 10, pp. 75-91, 1994.

[20] E. M. Costa-Neto and J. G. W. Marques, "Faunistic resources used as medicines by artisanal fishermen from Siribinha Beach, State of Bahia, Brazil," Journal of Ethnobiology, vol. 20, pp. 93-109, 2000.

[21] A. L. Silva, "Animais medicinais: conhecimento e uso entre as populações ribeirinhas do Rio Negro, Amazonas, Brasil. Boletim do Museu Paraense Emílio Goeldi," Série Ciências Naturais, vol. 3, pp. 343-357, 2008.

[22] T. M. Brooks, R. A. Mittermeier, C. G. Mittermeier et al., "Habitat loss and extinction in the hotspots of biodiversity," Conservation Biology, vol. 16, no. 4, pp. 909-923, 2002.

[23] I. R. Leal, J. M. C. Da Silva, M. Tabarelli, and T. E. Lacher Jr., "Changing the course of biodiversity conservation in the caatinga of northeastern Brazil," Conservation Biology, vol. 19, no. 3, pp. 701-706, 2005.
[24] R. R. N. Alves, H. N. Lima, M. C. Tavares, W. M. S. Souto, R. R. D. Barboza, and A. Vasconcellos, "Animal-based remedies as complementary medicines in Santa Cruz do Capibaribe, Brazil," BMC Complementary and Alternative Medicine, vol. 8, article 44, 2008.

[25] E. L. Araújo, C. C. Castro, and U. P. Albuquerque, "Dynamics of Brazilian Caatinga a review concerning the plants, environment and people," Functional Ecosystems and Communities, vol. 1, pp. 15-29, 2007.

[26] U. P. de Albuquerque, P. M. de Medeiros, A. L. S. de Almeida et al., "Medicinal plants of the caatinga (semi-arid) vegetation of NE Brazil: a quantitative approach," Journal of Ethnopharmacology, vol. 114, no. 3, pp. 325-354, 2007.

[27] R. R. N. Alves, "Fauna used in popular medicine in Northeast Brazil," Journal of Ethnobiology and Ethnomedicine, vol. 5, article 1, 2009.

[28] R. R. N. Alves, L. E. T. Mendonça, M. V. A. Confessor, W. L. S. Vieira, and L. C. S. Lopez, "Hunting strategies used in the semi-arid region of northeastern Brazil," Journal of Ethnobiology and Ethnomedicine, vol. 5, article 12, 2009.

[29] S. Rastogi and K. Kaphle, "Sustainable traditional medicine: taking the inspirations from ancient veterinary science," Evidence-Based Complementary and Alternative Medicine, vol. 5, pp. 1-6, 2008.

[30] W. E. G. Muller, R. Batel, H. C. Schroder, and I. M. Muller, "Traditional and modern biomedical prospecting: part Ithe history sustainable exploitation of biodiversity (sponges and invertebrates) in the Adriatic Sea in Rovinj (Croatia)," Evidence-Based Complementary and Alternative Medicine, vol. 1, pp. 171-182, 2004.

[31] W. E. G. Muller, H. C. Schroder, M. Wiens, S. P. Ottstadt, R. Batel, and I. M. Muller, "Traditional and modern biomedical prospecting: part II-the benefits approaches for a sustainable exploitation of biodiversity (secondary metabolites and biomaterials from sponges)," Evidence-Based Complementary and Alternative Medicine, vol. 1, pp. 133-144, 2004.

[32] E. L. Cooper, "Bioprospecting: a CAM frontier," EvidenceBased Complementary and Alternative Medicine, vol. 2, no. 1, pp. 1-3, 2005.

[33] E. L. Cooper, "CAM, eCAM, bioprospecting: the 21st century pyramid," Evidence-Based Complementary and Alternative Medicine, vol. 2, no. 2, pp. 125-127, 2005.

[34] United Nations Development Programme, "Atlas do Desenvolvimento Humano no Brasil," January 2009, http://www .pnud.org.br/atlas/.

[35] H. P. Huntington, "Using traditional ecological knowledge in science: methods and applications," Ecological Applications, vol. 10, no. 5, pp. 1270-1274, 2000.

[36] Centro Brasileiro de Classificação de Doenças, "Classificação e Estatística Internacional de Doenças e Problemas Relacionados à Saúde," February 2009, http://www.datasus.gov .br/cid10/webhelp/cid10.htm.

[37] M. Heinrich, A. Ankli, B. Frei, C. Weimann, and O. Sticher, "Medicinal plants in Mexico: healers' consensus and cultural importance," Social Science and Medicine, vol. 47, no. 11, pp. 1859-1871, 1998.

[38] O. Phillips, A. H. Gentry, C. Reynel, P. Wilkin, and B. C. Galvez-Durand, "Quantitative ethnobotany and Amazonian conservation," Conservation Biology, vol. 8, no. 1, pp. 225-248, 1994.

[39] R. R. D. Barboza, W. M. S. Souto, and J. S. Mourão, "The use of zootherapeutics in folk veterinary medicine in the district of Cubati, Paraíba State, Brazil," Journal of Ethnobiology and Ethnomedicine, vol. 3, article 32, 2007. 
[40] C. De Fátima, C. B. R. De Almeida, and U. P. De Albuquerque, "Uso e conservação de plantas e animais medicevais no estado de pernambuco (nordeste do Brasil): um estudo de caso," Interciencia, vol. 27, no. 6, pp. 276-285, 2002.

[41] L. Apaza, R. Godoy, D. Wilkie et al., "Markets and the use of wild animals for traditional medicine: a case study among the Tsimane' Amerindians of the Bolivian rain forest," Journal of Ethnobiology, vol. 23, pp. 47-64, 2003.

[42] M. L. V. Silva, A. G. C. Alves, and A. V. Almeida, "A zooterapia no Recife (Pernambuco): uma articulação entre as práticas e a história," Biotemas, vol. 17, pp. 95-116, 2004.

[43] R. R. N. Alves, "Use of marine turtles in zootherapy in Northeast Brazil," MTN, vol. 112, pp. 16-17, 2006.

[44] L. N. Kakati, A. Bendang, and V. Doulo, "Indigenous knowledge of zootherapeutic use of vertebrate origin by the Ao tribe of Nagaland," The Journal of Human Ecology, vol. 19, pp. 163167, 2006.

[45] M. M. Mahawar and D. P. Jaroli, "Animals and their products utilized as medicines by the inhabitants surrounding the Ranthambhore National Park, India," Journal of Ethnobiology and Ethnomedicine, vol. 2, article 46, 2006.

[46] R. R. N. Alves and I. L. Rosa, "Zootherapy goes to town: the use of animal-based remedies in urban areas of NE and N Brazil," Journal of Ethnopharmacology, vol. 113, no. 3, pp. 541-555, 2007.

[47] E. M. Costa-Neto, "Healing with animals in Feira de Santana City, Bahia, Brazil," Journal of Ethnopharmacology, vol. 65, no. 3, pp. 225-230, 1999.

[48] R. R. N. Alves and G. G. Santana, "Use and commercialization of Podocnemis expansa (Schweiger 1812) (Testudines: Podocnemididae) for medicinal purposes in two communities in North of Brazil," Journal of Ethnobiology and Ethnomedicine, vol. 4, article 3, 2008.

[49] R. R. N. Alves and I. L. Rosa, "Use of tucuxi dolphin Sotalia fluviatilis for medicinal and magic/ religious purposes in north of Brazil," Human Ecology, vol. 36, no. 3, pp. 443-447, 2008.

[50] R. R. N. Alves and G. A. Pereira-Filho, "Commercialization and use of snakes in North and Northeastern Brazil: implications for conservation and management," Biodiversity and Conservation, vol. 16, no. 4, pp. 969-985, 2007.

[51] A. V. Almeida, "Prescrições zooterápicas indígenas brasileiras nas obras de Guilherme Piso (1611-1679)," in Atualidades em Etnobiologia e Etnoecologia. Recife. Brazil, A. G. C. Alves, R. F. P. Lucena, and U. P. Albuquerque, Eds., pp. 47-60, Sociedade Brasileira de Etnobiologia e Etnoecologia, Nuppea, Brazil, 2005.

[52] E. L. Cooper, "Complementary and alternative medicine, when rigorous, can be science," Evidence-Based Complementary and Alternative Medicine, vol. 1, pp. 1-4, 2004.

[53] P. Roch, "What can we learn from marine invertebrates to be used as complementary antibiotics?" in Complementary and Alternative Approaches to Biomedicine, E. L. Cooper and N. Yamaguchi, Eds., pp. 391-404, Kluwer Academic/Plenum Publishers, New York, NY, USA, 2004.

[54] E. L. Cooper, B. Ru, and N. Weng, "Earthworms: sources of antimicrobial and anticancer molecules," in Complementary and Alternative Approaches to Biomedicine, E. L. Cooper and N. Yamaguchi, Eds., pp. 359-390, Kluwer Academic/Plenum Publishers, New York, NY, USA, 2004.

[55] P. Roch, A. Beschin, and E. Bernard, "Antiprotozoan and antiviral activities of non-cytotoxic truncated and variant analogues of mussel defensin," Evidence-Based Complementary and Alternative Medicine, vol. 1, pp. 167-174, 2004.

[56] T. McGirk, “Gene piracy," Time, vol. 1, pp. 20-25, 1998.
[57] I. Amato, "From 'hunter magic', a pharmacopeia?" Science, vol. 258, no. 5086, p. 1306, 1992.

[58] L. H. Lazarus and M. Attila, "The toad, ugly and venomous, wears yet a precious jewel in his skin," Progress in Neurobiology, vol. 41, no. 4, pp. 473-507, 1993.

[59] M. Hamada and T. Nagai, "Inorganic components of bones of fish and their advanced utilization," Journal of Shimonoseki University of Fisheries, vol. 43, pp. 185-194, 1995.

[60] C. W. Finkl, "Os medicamentos do mar," in Enciclopédia dos Mares, J. Y. Cousteau, Ed., pp. 74-75, Salvat, Rio de Janeiro, Brazil, 1984.

[61] S. Adeodato, "Os santos remédios do mar," Globo Ciência, pp. 20-25, 1997.

[62] R. Salte, K. Norberg, and O. R. Ødegaard, "Evidence of a protein C-like anticoagulant system in bony fish," Thrombosis Research, vol. 83, no. 5, pp. 389-397, 1996.

[63] R. Root-Bernstein and M. Root-Bernstein, Honey, Mud, Maggots and Other Medical Marvels: The sciences behind Folks Remedies and Old Wives' Tales, Houghton Mifflin Company, Boston, Mass, USA, 1999.

[64] R. Rubin, "Maggots and leeches: good medicine," January 2009, http://www.usatoday.com/news/health/2004-0707-eches-maggots_x.htm.

[65] J. Still, "Use of animal products in traditional Chinese medicine: environmental impact and health hazards," Complementary Therapies in Medicine, vol. 11, no. 2, pp. 118-122, 2003.

[66] R. R. N. Alves, N. A. L. Neto, S. E. Brooks, and U. P. Albuquerque, "Commercialization of animal-derived remedies as complementary medicine in the semi-arid region of Northeastern Brazil," Journal of Ethnopharmacology, vol. 124, no. 3, pp. 600-608, 2009.

[67] R. D. Simpson, R. A. Sedjo, and J. W. Reid, "Valuing biodiversity for use in pharmaceutical research," Journal of Political Economy, vol. 104, no. 1, pp. 163-185, 1996.

[68] S. K. Murari, F. J. Frey, B. M. Frey, T. V. Gowda, and B. S. Vishwanath, "Use of Pavo cristatus feather extract for the better management of snakebites: neutralization of inflammatory reactions," Journal of Ethnopharmacology, vol. 99, no. 2, pp. 229-237, 2005.

[69] N. G. Bisset, "One man's poison, another man's medicine?” Journal of Ethnopharmacology, vol. 32, no. 1-3, pp. 71-81, 1991.

[70] V. Brazil, "Do emprego da peçonha em terapêutica," Biologia Médica São Paulo, vol. 1, pp. 7-21, 1934.

[71] M. Hayman and D. I. Macht, "Clinical and biochemical studies in cobra venom therapy," Medical Record, vol. 152, pp. 67-69, 1940.

[72] G. G. Habermehl, "Therapeutic use of animal venoms," in Venomous Animals and Their Toxins, G. G. Habermehl, Ed., pp. 184-185, Springer, Berlin, Germany, 1981.

[73] R. Chen and S. E. Robinson, "The effect of cholinergic manipulations on the analgesic response to cobrotoxin in mice," Life Sciences, vol. 47, no. 21, pp. 1949-1954, 1990.

[74] X. C. Pu, P. T. H. Wong, and P. Gopalakrishnakone, "A novel analgesic toxin (Hannalgesin) from the venom of king cobra (Ophiophagus hannah)," Toxicon, vol. 33, no. 11, pp. 14251431, 1995.

[75] A. Mulu, B. Tessema, and F. Derbie, "In vitro assessment of the antimicrobial potential of honey on common human pathogens," The Ethiopian Journal of Health Development, vol. 18 , pp. 107-112, 2004. 
[76] T.-J. Park, J.-S. Kim, S.-S. Choi, and Y. Kim, "Cloning, expression, isotope labeling, purification, and characterization of bovine antimicrobial peptide, lactophoricin in Escherichia coli," Protein Expression and Purification, vol. 65, no. 1, pp. 2329, 2009.

[77] R. Somvanshi, "Veterinary medicine and animal keeping in ancient India," Asian Agri-History, vol. 10, no. 2, pp. 133-146, 2006.

[78] R. R. N. Alves, G. A. P. Filho, and Y. C. C. De Lima, "Snakes used in ethnomedicine in Northeast Brazil," Environment, Development and Sustainability, vol. 9, no. 4, pp. 455-464, 2007.

[79] Instituto Brasileiro do Meio Ambiente e dos Recursos Naturais Renováveis (IBAMA), "Lista das Espécies da Fauna Brasileira Ameaçadas de Extinção," January 2009, http://www.ibama.gov.br/.

[80] Ministério do Meio Ambiente (MMA), "Lista Nacional de Espécies de Invertebrados Aquáticos e Peixes Sobreexplotadas ou ameaçadas de Sobreexplotação," http://www.prpe.mpf.gov .br/internet/content/download/2830/22487/file/in052004mma.pdf.

[81] C. Anyinam, "Ecology and ethnomedicine: exploring links between current environmental crisis and indigenous medical practices," Social Science and Medicine, vol. 40, no. 3, pp. 321329, 1995.

[82] B. Saad, H. Azaizeh, and O. Said, "Tradition and perspectives of Arab herbal medicine: a review," Evidence-Based Complementary and Alternative Medicine, vol. 2, no. 4, pp. 475-479, 2005.

[83] H. Azaizeh, B. Saad, E. Cooper, and O. Said, "Traditional arabic and islamic medicine, a Re-emerging health aid," Evidence-Based Complementary and Alternative Medicine, vol. 7, no. 4, pp. 419-424, 2010.

[84] B. T. Shaikh and J. Hatcher, "Complementary and alternative medicine in Pakistan: prospects and limitations," EvidenceBased Complementary and Alternative Medicine, vol. 2, no. 2, pp. 139-142, 2005.

[85] R. R. N. Alves and I. L. Rosa, "Medicinal animals for the treatment of asthma in Brazil," Journal of Alternative and Complementary Medicine, vol. 14, no. 4, pp. 350-351, 2008.

[86] E. L. Cooper, "eCAM: an emerging linkage with ethnopharmacology?" Evidence-Based Complementary and Alternative Medicine, vol. 5, no. 4, pp. 365-366, 2008.

[87] R. R. N. Alves and I. M. L. Rosa, "Biodiversity, traditional medicine and public health: where do they meet?" Journal of Ethnobiology and Ethnomedicine, vol. 3, article 14, 2007.

[88] R. R. N. Alves, C. C. Silva, R. R. D. Barboza, and M. S. W. Souto, "Zootherapy as an alternative therapeutic in South America," Journal of Alternative Medicine Research, vol. 1, pp. 21-47, 2009.

[89] F. S. Ferreira, S. V. Brito, S. C. Ribeiro, W. O. Almeida, and R. R. N. Alves, "Zootherapeutics utilized by residents of the community Poço Dantas, Crato-CE, Brazil," Journal of Ethnobiology and Ethnomedicine, vol. 5, article 21, 2009. 


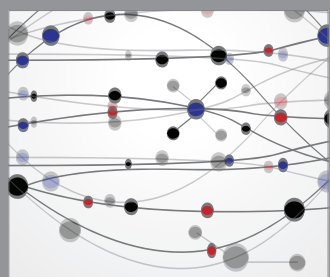

The Scientific World Journal
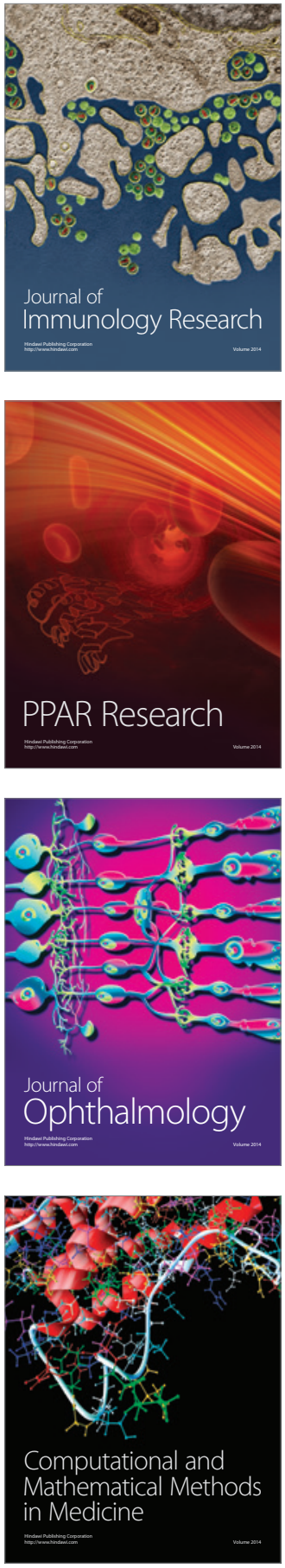

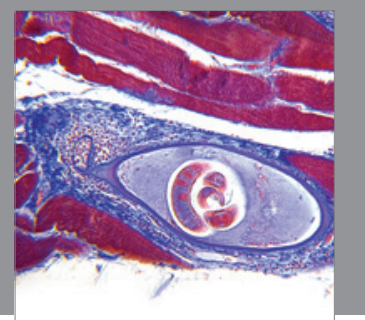

Gastroenterology

Research and Practice
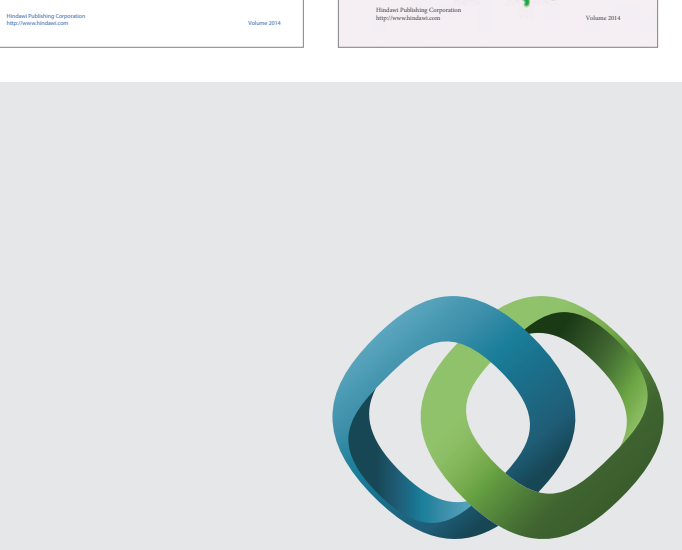

\section{Hindawi}

Submit your manuscripts at

http://www.hindawi.com
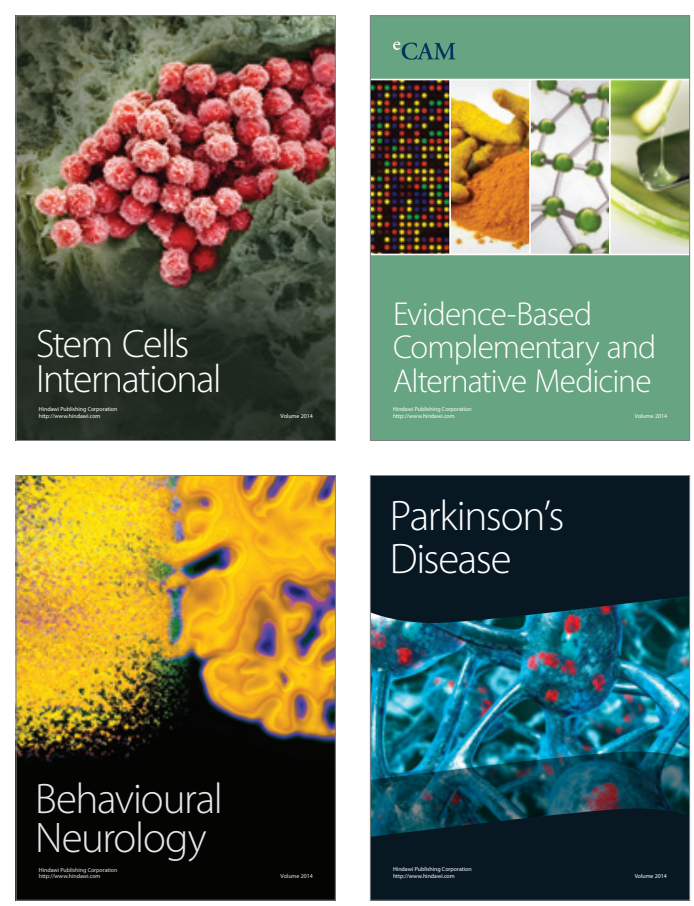

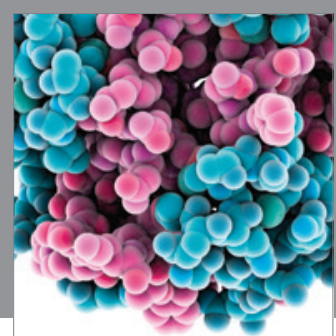

Journal of
Diabetes Research

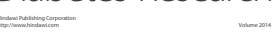

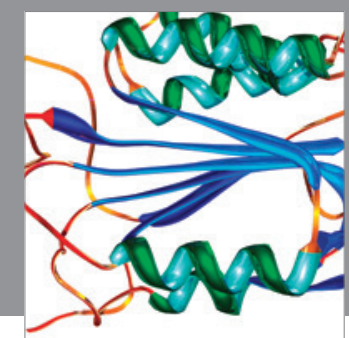

Disease Markers
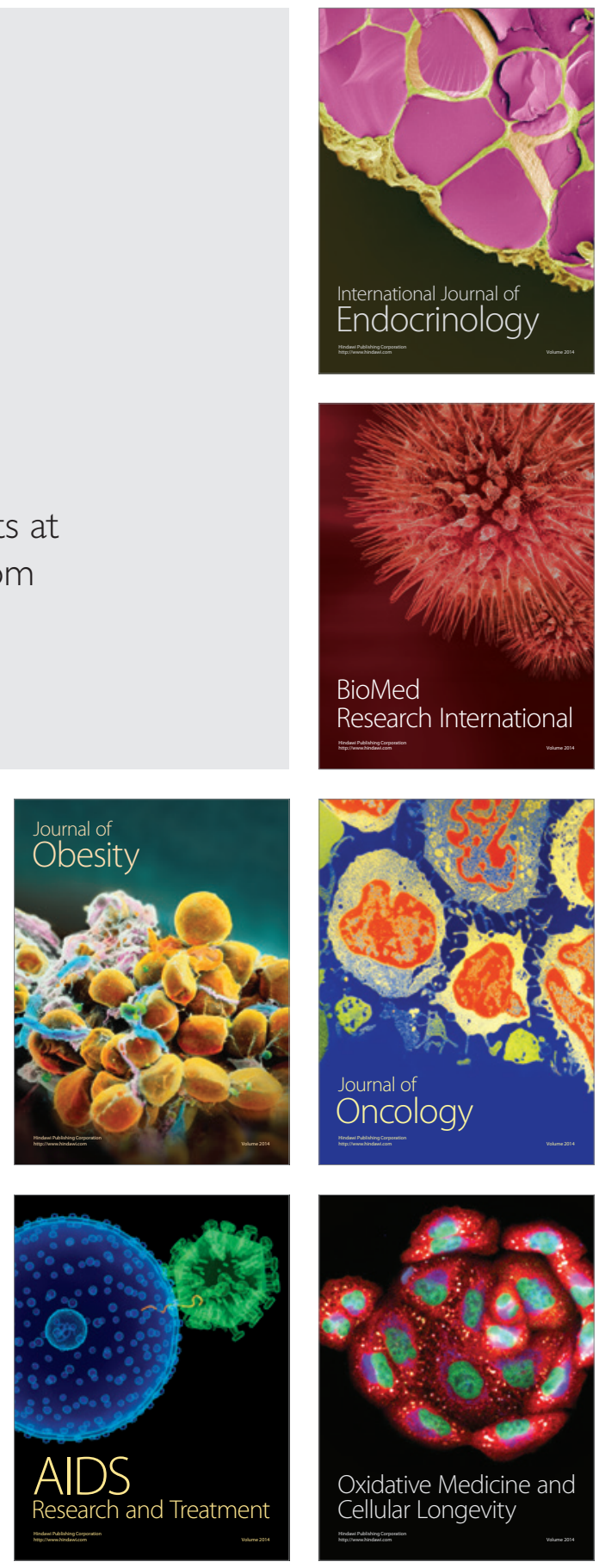